\title{
Focusing on the
}

\section{ubiquitin-proteasome system}

If you are a molecular or cell biologist, chances are that at some stage you will encounter some aspect of the ubiquitin-proteasome system. Although relatively young, the field's exponential growth over the last decade continues unabated and it is now clear that regulated protein degradation by the ubiquitin-proteasome system affects practically every cellular process. Its significance was rightly acknowledged last year with the Nobel Prize in Chemistry (http://www.nature.com/ncb/journal/v6/n11/full/ncb11041011.html). The discovery of non-proteolytic functions for this pathway has further cemented its pivotal place in cell physiology.

Following the discovery of ubiquitin in the late 1970s and the delineation of the enzymology of the pathway, its regulatory potential for orchestrating complex cellular programmes was initially severely underestimated. In those days, proteolysis took a back seat while gene expression and signal transduction held centre stage as the dominant regulatory mechanisms. Studies on cell-cycle progression provided some of the earliest insights into the regulatory power of this pathway (again marked by a Nobel Prize in 2001). Although it is now evident that the ubiquitin-proteasome pathway affects many processes, proteolysis is most fundamentally entwined with the cell cycle - probably because a series of absolute decisions is required and the synergy between these two areas of research remains vibrant.

In this issue, we are pleased to present a collection of specially commissioned articles that discuss recent developments and emerging themes in this complex and dynamic area. We hope that this series serves to bring both the expert and the novice up to date.

Perhaps, with the luxury of hindsight, the fact that proteolysis directs so many cellular processes may not seem all that surprising. After all, proteolysis can rapidly and irreversibly destroy proteins; it can thereby dismantle existing cellular programmes and allow new ones to take root. More surprising is the description of ubiquitin functions that go considerably beyond tagging proteins for degradation; for example, it is now fully appreciated that ubiquitin is a versatile signalling molecule. Tagging by mono-ubiquitin and ubiquitin chains have non-proteolytic roles in membrane transport, chromatin structure and transcription, DNA repair and diverse signalling pathways. In his review, Zhijian Chen highlights proteolytic and non-proteolytic control of the NF- $\mathrm{KB}$ signalling pathway - a pathway in which ubiquitin exercises multiple levels of control - by ubiquitin and its relative, SUMO. The route to protein degradation was believed to be well charted, but it now turns out to be more circuitous than previously thought. This is apparent from Suzanne Elsasser and Daniel Finley's discussion of the emerging complexities of how substrates are targeted to the $26 \mathrm{~S}$ proteasome, and the surprising similarities between this targeting and how ubiquitinated substrates are ferried to Cdc48 - a multi-subunit ATPase complex involved in ubiquitindependent degradation, vesicle fusion and mitotic spindle assembly.

Another unanticipated finding is the degree of diversity among ubiquitin pathway enzymes, such as ligases and deubiquitinating enzymes, and among ubiquitin-like proteins. As ubiquitin's functions have diversified, so too have the domains that are specialized for recognizing ubiquitin in its many manifestations. So, it is now possible to schematize detailed networks of ubiquitin-based interactions in different processes. What better way to get a handle on the variety and complexity of pathway components than largescale proteomics and genomics? In their review, Steven Gygi and colleagues discuss new advances in mass spectrometry and the growing contributions of this approach to developing a comprehensive functional understanding of the ubiquitin-proteasome system.

Two articles by Thomas Sommer and colleagues and Judith Frydman and colleagues describe how the ubiquitin-proteasome system disposes of aberrant proteins at the endoplasmic reticulum and in the cytoplasm, respectively. Although ridding the cell of junk proteins has been one of the earliest recognized functions of this pathway, it is clear that many key questions remain; for example, how misfolded proteins are recognized, how they are eliminated from the endoplasmic reticulum into the cytoplasm, and how chaperones - better known for their role in protein folding - might also direct proteolysis.

Finally, Jonathon Pines and Catherine Lindon remind us that there is a time and a place for everything, including protein degradation. The importance of temporal regulation in this pathway has been appreciated for some time, but the significance of spatial control has yet to be fully explored. As the authors discuss, the 'where' of protein ubiquitination and degradation will surely emerge to be as important as the 'when'.

Of course, this collection of articles is not meant to provide comprehensive coverage of the field; instead, we have attempted to cover some particularly topical and emerging issues. We are pleased to acknowledge the financial support of Millennium Pharmaceuticals and Boston Biochem in producing this Focus. As always, Nature Cell Biology has sole responsibility for editorial content. These Focus articles are freely available for six months and, in addition, are hosted at a dedicated website (http://www.nature.com/ncb/webfocus/ubiquitin), which also includes a library of selected articles on this topic from across the Nature research and review journals. Articles in this library will be freely available for three months. Finally, as always your feedback is welcome and you can send us your comments via a link on the Focus website. 